\title{
Predictors of a successful "crosstalk" ablation technique during second-generation cryoballoon ablation in patients with atrial fibrillation
}

\author{
Tatsuhiko Hirao $^{1}$, Yasuteru Yamauchi ${ }^{1}$, Rena Nakamura ${ }^{1}$, Takatoshi Shigeta ${ }^{1}$, Hiroshi \\ Yoshida $^{1}$, Shinichi Tachibana ${ }^{1}$, Atsuhito Oda ${ }^{1}$, Aki Ito ${ }^{1}$, Mitsutoshi Asano ${ }^{1}$, Hidetoshi \\ Suzuki $^{1}$, Tsukasa Shimura ${ }^{1}$, Manabu Kurabayashi ${ }^{1}$, Masahiko Goya ${ }^{2}$, Kaoru Okishige ${ }^{1}$, and \\ Tetsuo Sasano ${ }^{2}$ \\ ${ }^{1}$ Yokohama City Minato Red Cross Hospital \\ ${ }^{2}$ Tokyo Medical and Dental University
}

August 10, 2020

\begin{abstract}
Introduction: The "crosstalk" (CST) ablation technique has been reported to reduce unnecessary ablation during cryoballoon (CB) ablation (CBA). Nevertheless, it is unclear which situations will necessitate the adoption of the technique. Methods and Results: The effect of the technique was analyzed in AF patients underwent CBA from July 2017 to February 2020. The balloon occlusion status and nadir temperature (NT) were compared, and all ablated PVs were categorized into three groups according to the necessity and effectiveness of the technique. Of 1082 superior PVs (SPVs), 16, 40, and 1026 were identified in the CST success group, CST failure group, and control group, respectively. The proportion of SPVs ablated with complete occlusion with CB was significantly higher in the CST success group (100\%) than in the CST failure group (16.7\%) or control group (49.4\%) (CST success group vs. CST failure group, $\mathrm{p}<0.001$; CST success group vs. control group, $\mathrm{p}<0.002$ ). The proportion of SPVs ablated with NT [?]- $46^{\circ} \mathrm{C}$ was higher in the CST success group (100\%) than in the CST failure group $(56.7 \%)(\mathrm{p}<0.05)$. The CST ablation technique was always effective if CBA of the SPVs was performed with both complete occlusion and NT [?]-46degC and was almost always ineffective if it did not meet these two criteria (sensitivity, 100\%; specificity, 93\%). Conclusion: Successful CST ablation was highly predicted if complete PV occlusion and NT [?]-46degC during CBA of the SPVs were achieved, which could be useful when adopting the technique targeting inferior PVs to reduce unnecessary freezing during SPV isolation.
\end{abstract}

\section{KEYWORDS}

atrial fibrillation, catheter ablation, crosstalk technique, cryoballoon, electrical connection, pulmonary vein isolation

\section{INTRODUCTION}

With respect to efficacy, cryoballoon (CB)-guided pulmonary vein (PV) isolation of paroxysmal atrial fibrillation $(\mathrm{AF})$ can provide a comparable ${ }^{1-4}$ or better outcome than contact force-guided radiofrequency catheter ablation. ${ }^{5}$ Thus, CB ablation is clearly one of the most effective treatment modalities for AF. ${ }^{6,7}$ However, $\mathrm{CB}$ application connotes the risk of severe PV stenosis/occlusions, ${ }^{8,9}$ and may damage to the extracardiac structures such as the esophagus, ${ }^{10}$ phrenic nerve, and bronchus. ${ }^{11,12}$

Chun et al. reported the "crosstalk" (CST) phenomenon in which CB application of the left inferior PV (LIPV) was able to ablate the gap sites along the inferior aspect of the left superior PV (LSPV) when 
applications failed to isolate the LSPV. ${ }^{13}$ This CST ablation technique can be beneficial in terms of reducing unnecessary freezing of the atrial tissue. Nevertheless, it is unclear which situations will necessitate the adoption of the CST ablation technique. Miyazaki et al. showed that the CST ablation technique was useful in cases in which the LSPV could not be isolated even if complete PV occlusion was achieved with the CB, and the nadir balloon temperature (NT) of the CB was not fully analyzed. ${ }^{14}$

The present study aimed to investigate the relationship between the NT and occlusion status of the CB and the efficacy of CST ablation. Additionally, we sought to find the predictors of appropriate conditions for when the CST ablation technique should be adopted.

\section{METHODS}

\subsection{Study population}

Patients with drug-refractory AF who underwent de novo CB ablation using second-generation CB from July 2017 to February 2020 were included in this retrospective, single-center, non-randomized study. In order to establish the left atrial (LA) and PV anatomy, all patients underwent a computed tomography scan prior to ablation. All patients provided informed consent prior to the ablation procedure. We excluded those with left common PVs, huge PVs in which the CB was applied separately to the upper and lower parts of the PV, LA diameter $>55 \mathrm{~mm}$, intracardiac thrombi, uncontrolled thyroid dysfunction, contraindications for anticoagulation, and uncontrollable heart failure.

This study was conducted in accordance with the principles outlined in the Declaration of Helsinki. The study protocol was approved by the local institutional committee on human research at the Yokohama-City Bay Red Cross Hospital (approval number: 2017-81).

\subsection{Pre-ablation preparation protocol}

Prior to the procedure, all antiarrhythmic drugs were discontinued for at least 5 half-lives. Direct oral anticoagulants were changed to dabigatran from the day before the ablation. Patients on warfarin continued taking it during the peri-procedural period. An international normalized ratio level between 2.0 and 3.0 was considered acceptable. All patients continued taking anticoagulants throughout the peri-procedural period. Using prone-positional enhanced computed tomography or intracardiac/transesophageal echocardiography, we confirmed that there were no intracardiac thrombi before the ablation. Surface electrocardiograms and bipolar intracardiac electrograms filtered from 30 to $500 \mathrm{~Hz}$ were monitored.

Ablation was performed under general anesthesia with dexmedetomidine, thiopental, and rocuronium. The ablation procedure has been previously described in detail. ${ }^{15}$ Briefly, a standard transvenous approach was employed for transseptal puncture and subsequent PV isolation. A 7-Fr 20-pole three-site mapping catheter (BeeAT; Japan Lifeline, Tokyo, Japan) was located in the coronary sinus for anatomic guidance. With a radiofrequency needle (Baylis Medical, Montreal, QC, Canada), a single transseptal puncture of the fossa ovalis was performed using the Brockenbrough technique. The puncture was guided by intracardiac echocardiography (ACUSON AcuNav; Biosense Webster, Diamond Bar, CA, USA) and biplane fluoroscopy (right and left anterior oblique projections). Thereafter, heparin was administered intravenously to achieve an activated clotting time between 300 and 350 seconds during the ablation procedure.

\subsection{Cryoballoon ablation protocol}

After gaining LA access, we injected contrast into the LA to define the position of each PV ostium. Through a steerable 15-Fr sheath (FlexCath Advance; Medtronic, Minneapolis, MN, USA), an inner lumen mapping catheter (Achieve; Medtronic, Minneapolis, MN, USA) was advanced into each PV ostium. Subsequently, a 28-mm second-generation CB (Arctic Front Advance; Medtronic, Minneapolis, MN, USA) was advanced over the inner lumen mapping catheter, inflated, and positioned sequentially in the PV ostium of each vein. An optimal vessel occlusion was considered to be achieved upon selective contrast injection from the tip of the CB showing total contrast retention with no backflow into the LA. Once a complete occlusion was documented, we withdrew the CB slightly and allowed leakage around the PV-balloon interface to better 
define the PV ostium and ensured a proximal ablation position. We reapplied only the minimal amount of pressure needed to regain the occlusion before the ablation, and cryothermal energy delivery was commenced. Standard cryoenergy applications lasted for 180 seconds. If PV potentials were visible during freezing energy delivery, the time to PV isolation was recorded when the potentials completely disappeared or became dissociated from the LA activity.

When the superior PV was not isolated with CB application even if the PV was completely occluded, we introduced the CST ablation technique and advanced the CB into the ipsilateral inferior PV, while the superior PV potentials were monitored during freezing energy delivery by a Lasso catheter placed inside the superior PV. When the time to PV isolation was clearly measured, we used it as a guide in deciding the freezing duration; we usually added more than 120 seconds to it as long as the total freezing duration did not exceed 240 seconds. If the superior PV was isolated during CB ablation to the ipsilateral inferior PV, we regarded this as successful CST ablation. When PV isolation was not achieved with CB application only, we performed touch-up ablation of the PV using an open-irrigated radiofrequency catheter (FlexAbility and TactiCath; Abbott, Chicago, IL, USA or ThermoCool; Biosense Webster, Irvine, CA, USA). CB ablation was applied in the following order: LSPV, LIPV, right inferior PV, and right superior PV (RSPV).

During cryoablation of the right-sided PVs, continuous phrenic nerve stimulation with a decapolar catheter positioned in the superior vena cava was performed. The integrity of the phrenic nerve was monitored by intermittent fluoroscopy, tactile feedback, and right-sided compound motor action potential (CMAP) monitoring. When the CMAP amplitude decreased by more than $30 \%$ from the control value, CB application was prematurely terminated to avoid any injury to the phrenic nerve.

During CB ablation, the luminal esophageal temperature was also continuously monitored using an esophageal temperature probe (SensiTherm; Abbott, Chicago, IL, USA or Esophaster; Japan Lifeline, Tokyo, Japan). CB application was stopped when the esophageal temperature dropped below $15^{\circ} \mathrm{C}$.

\subsection{Statistical analysis}

Continuous variables are presented as mean \pm standard deviation or as median and interquartile range and were compared using the t-test or Wilcoxon rank-sum test based on the distribution. Categorical variables are expressed as number and percentage and were compared using the $\chi^{2}$ test. A $p<0.05$ was considered to indicate statistical significance. Multiple comparisons of continuous variables were performed using the Wilcoxon/Kruskal-Wallis test, whereas multiple comparisons of numerous variables were conducted with the $\chi^{2}$ test. If the test indicated a significant difference, we performed post-hoc analysis with adjustment using the Bonferroni method.

\section{RESULTS}

\subsection{Patient characteristics}

A total of 541 patients with AF (average age: $67.4 \pm 10.8$ years; $71 \%$, males) who underwent de novo catheter ablation using the second-generation CB were included in this study. The patients' baseline characteristics are summarized in Table 1.

\subsection{Classification of the PVs based on the outcome of CB ablation and CST ablation technique}

Out of 1082 PVs, 56 superior PVs (31 LSPVs and 25 RSPVs) were not isolated by CB ablation of the superior PV, and the CST ablation technique for a successful PV isolation was attempted. Among these 56 superior PVs, the CST ablation technique was effective in 16 PVs (CST success group) but ineffective in 40 PVs that required touch-up radiofrequency ablation to achieve PV isolation (CST failure group). The third group involved superior PVs that were isolated only with ablation targeting the superior PV ("unnecessary CST" group = control group). Thus, we categorized the ablated superior PVs into three groups according to the effects of CB ablation and CST ablation technique, as shown in Figure 1.

3.3 Comparisons of CB procedures among the CST success group, CST failure group, and control group 
Two factors of CB applications to the superior PVs - namely, NT and percentage of complete occlusionswere compared among the three groups (Figure 2). Differences in the NT among the three groups are illustrated in Figure 2A; the NT was significantly lower in the CST success and control groups than in the CST failure group $(\mathrm{p}<0.001)$. Of note, complete occlusion of the superior PVs with the CB was significantly more highly achieved in the CST success group than in the CST failure and control groups (Figure 2B).

We analyzed the NT distribution during incomplete and complete occlusion applications, as shown by the graph in Figure 3. The NT in the CST success group was mainly observed in the temperature range of [?]-46degC when complete occlusion was achieved. In contrast, the NT in the CST failure group was widely scattered throughout all temperatures, including those during an incomplete occlusion of the superior PVs and during complete occlusions other than those that were mainly observed in the CST success group. As suggested by this graph, the CST ablation technique was always effective if CB ablation of the superior PVs was performed with both complete occlusion and NT [?]-46degC and was almost always ineffective if it did not meet these two criteria (sensitivity, 100\%; specificity, 93.8\%). When only complete PV occlusion was used as the criterion, the sensitivity and specificity were $93.8 \%$ and $87.5 \%$, respectively, which were worse than those of the abovementioned criteria.

\subsection{Characteristics of the CB procedure in the CST success group}

The characteristics of parameters during CB applications to the superior and inferior PVs in 16 cases in the CST success group are summarized in Table 2. Complete occlusion of the superior PVs with the CB was achieved in 15/16 cases (93.8\%) and the minimum NT for CB application to the superior PVs ranged from -46degC to -60degC. In all 16 cases except case 9, the first CB application to the inferior PVs succeeded in isolating the superior PVs and no further applications were added. In case 9, because the esophageal temperature during the first $\mathrm{CB}$ application to the inferior PVs decreased below 15degC, the application was ceased and superior PV isolation was achieved by the next application that was added after the esophageal temperature recovered to above $30 \operatorname{deg}$.

\subsection{Case presentation of a successful CST ablation technique}

This case was a patient with paroxysmal AF who underwent de novoablation with a CB (Figure 3). The LSPV was not isolated after the first CB application with an NT of -52degC and complete LSPV occlusion (Figure 4A). We did not add a CB application to the LSPV but instead attempted LIPV ablation. At 21 seconds after initiating the ablation, both the LSPV and LIPV were simultaneously isolated (Figure 4B). Furthermore, frequent LIPV ectopic beats, which conducted to the LSPV without conducting to the LA, were observed. These electrophysiological findings could not be explained without the presence of an intervening connection between the LSPV and LIPV.

\section{DISCUSSION}

The major finding of this study was that in cases wherein CB ablation of the superior PVs was unable to isolate the PVs, the CST ablation technique was highly successful if CB ablation of the superior PVs met the following two criteria: NT [?]-46degC and complete occlusion of the superior PVs. Furthermore, the CST ablation technique was mostly ineffective in cases wherein these two criteria were not met.

\subsection{Factors for an effective CST ablation}

During CST ablation for PV isolation, PV potentials sometimes remain even if the targeted PV is perfectly occluded and the NT is low enough to freeze the PV ostium. Following the recognition of the CST phenomenon by Chun et al., ${ }^{13}$ the CST ablation technique was introduced into the ablation setting. Previous studies reported that complete occlusion of the superior PVs was one of the proposed factors for an effective CST ablation; however, it was not statistically analyzed. ${ }^{13,14}$ By comparing the ablation data among the CST success, failure, and control groups, we were able to obtain the sensitivity and specificity of factors for a successful CST ablation and proposed two highly predictable factors for CST ablation - namely, NT [?]-46degC and complete PV occlusion during CB ablation of the superior PVs. 


\subsection{Clinical utility of the CST ablation technique}

There can be an alternative to adding more $\mathrm{CB}$ applications to the present $\mathrm{PV}$ or shifting the CB to the ipsilateral PV in expectation of a CST phenomenon in cases with electrical connections remaining between the LA and PV despite rigorous $\mathrm{CB}$ applications to the superior PV. The success rate of PV isolation using the CST ablation technique was reported by Miyazaki et al. ${ }^{14}$ to be $41.9 \%$ (13/31 cases) and was $48.4 \%$ (15/31 patients) in our study, which is relatively high. Therefore, once the predictors postulated in this study could be introduced into the CB ablation setting, superior PV isolation would be expected to be more efficiently achieved, which would enable a reduction in the ablation application time and freezing damage to the myocardium and collateral tissues, including the esophagus and phrenic nerve. To the best of our knowledge, there has been no systematic study on how often the CST ablation technique can be applied in actual CB ablation. Nevertheless, the occurrence rate of successful CST cases among all CB ablation cases was $2.9 \%$ (16/541 patients) in our study and was reported to be $2.9 \%(15 / 511$ patients $)$ and $4.7 \%(32 / 676$ patients) in previous studies, respectively. ${ }^{2,14}$

\subsection{The mechanisms of the CST phenomenon}

The two distinct time differences required for the successful isolation of the superior and ipsilateral inferior PVs in this study would lead to the possibility of two mechanisms for the CST phenomenon. As shown in Table 2, the isolated cases were divided into two groups. In one group, an almost simultaneous isolation of both PVs was observed in cases 10, 14, and 15 ("simultaneous" group), whereas in another group, the superior PV was isolated with a non-simultaneous but rather different timing from that of the ipsilateral inferior PV, as in cases 7, 9, 11, and 16 ("non-simultaneous" group). Based on these findings, two mechanisms could be proposed, as delineated in Figure 5. An epicardial connection intervening between the ipsilateral PVs (epicardial conduction) may exist in the "simultaneous" group. On the other hand, the thick carina tissue, which could interrupt a complete isolation of the superior PV by CB application to the superior PV until additional freezing has been applied to the inferior PV (carina conduction), may connect the superior and inferior PVs in the "non-simultaneous" group.

The prevalence of an epicardial conduction was reported by previous studies to be $13.5 \%-20 \%$. ${ }^{16-18}$ To date, various epicardial conduction paths between the PVs and other atrial structures have been reported: LSPVposterior LA, LSPV-vein of Marshall (VOM) area, LSPV-LA roof, left common PV (LCPV)-VOM area, LCPV-posterior LA, RSPV-posterior LA, and RSPV-LA roof. ${ }^{16-21}$ Takahashi et al. reported that out of 49 cases in which the LSPV was difficult to isolate by radiofrequency catheter ablation, 7 cases had an earliest activation site in the LIPV during intra-LSPV pacing, which suggested an epicardial conduction between the ipsilateral PVs. ${ }^{20}$

The durability of acute PV isolation and the long-term AF survival rate have been reported to be lower in cases with epicardial conduction than in cases without such conduction. ${ }^{16-17}$ In our study, no acute electrical reconnections of the PVs were documented in the CST success group.

Anatomical reports have described that the thickest myocardial sleeves around the PVs are found in the carina region. ${ }^{22,23}$ Cabrera et al. reported these connections from an anatomical aspect. ${ }^{23,24}$ In histologic sections of 15 hearts, $27 \%, 53 \%$, and $20 \%$ of myocardial strands between the superior and inferior PVs were epicardial, subendocardial, and from both aspects of the PV wall, respectively. The strands on the epicardial PV wall are likely to correspond to electrical epicardial intervening connections. That anatomical aspect of the PVs may account for the two conduction patterns observed during CB applications and eventual CST phenomenon.

\subsection{Study limitations}

In our study population, the prevalence of successful CST cases was about $3 \%$ and the CST ablation technique was effective in 16 patients, which are small and may not have been adequate to derive any accurate factors for predicting a successful CST ablation. Additionally, whether the two factors derived could be useful predictors in the $\mathrm{CB}$ ablation setting was not evaluated owing to the retrospective non-randomized design 
of this study. Hence, these two factors should be evaluated in a prospective, randomized, multicenter study in the future.

\section{CONCLUSION}

A successful CST ablation technique was highly predicted if the NT was [?]-46degC and a complete PV occlusion during CB ablation of the superior PVs was achieved. The CST ablation technique targeting the ipsilateral inferior PV to reduce any unnecessary freezing during superior PV isolation could be useful to adopt.

\section{ACKNOWLEDGEMENTS}

We thank Mr. John Martin for his linguistic support.

\section{REFERENCES}

1. Kuck KH, Brugada J, Furnkranz A, et al. Cryoballoon or radiofrequency ablation for paroxysmal atrial fibrillation. N Engl J Med 2016;374(23):2235-2245.

2. Yang JD, Sun Q, Guo XG, et al. "Crosstalk" technique: a comparison between two generations of cryoballoon catheter. Pacing Clin Electrophysiol 2018;41(6):635-642.

3. Mortsell D, Arbelo E, Dagres N, et al. Cryoballoon vs. radiofrequency ablation for atrial fibrillation: a study of outcome and safety based on the ESC-EHRA atrial fibrillation ablation long-term registry and the Swedish catheter ablation registry. Europace 2019;21(4):581-589.

4. Murray MI, Arnold A, Younis M, Varghese S, Zeiher AM. Cryoballoon versus radiofrequency ablation for paroxysmal atrial fibrillation: a meta-analysis of randomized controlled trials. Clin Res Cardiol 2018;107(8):658-669.

5. Aryana A, Singh SM, Kowalski M, et al. Acute and long-term outcomes of catheter ablation of atrial fibrillation using the second-generation cryoballoon versus open-irrigated radiofrequency: a multicenter experience. J Cardiovasc Electrophysiol 2015;26(8):832-839.

6. Neumann T, Vogt J, Schumacher B, et al. Circumferential pulmonary vein isolation with the cryoballoon technique results from a prospective 3-center study. J Am Coll Cardiol 2008;52(4):273-278.

7. Knight BP, Novak PG, Sangrigoli R, et al. Long-term outcomes after ablation for paroxysmal atrial fibrillation using the second-generation cryoballoon: final results from STOP AF Post-Approval Study. JACC Clin Electrophysiol 2019;5(3):306-314.

8. Tokutake K, Tokuda M, Ogawa T, Matsuo S, Yoshimura M, Yamane T. Pulmonary vein stenosis after second-generation cryoballoon ablation for atrial fibrillation. HeartRhythm Case Rep 2017;3(1):36-39.

9. Watanabe K, Nitta J, Sato A, Goya M, Isobe M, Hirao K. Hemoptysis after five months of cryoballoon ablation: what is the relationship? HeartRhythm Case Rep 2017;3(7):357-359.

10. John RM, Kapur S, Ellenbogen KA, Koneru JN. Atrioesophageal fistula formation with cryoballoon ablation is most commonly related to the left inferior pulmonary vein. Heart Rhythm 2017;14(2):184-189.

11. Verma N, Gillespie CT, Argento AC, et al. Bronchial effects of cryoballoon ablation for atrial fibrillation. Heart Rhythm 2017;14(1):12-16.

12. Schweigert M, Almeida AB. Life-threatening pulmonary haemorrhage during cryoballoon ablation for atrial fibrillation. Eur J Cardiothorac Surg 2018;54:789-791.

13. Chun KR, Schmidt B, Metzner A, et al. The 'single big cryoballoon' technique for acute pulmonary vein isolation in patients with paroxysmal atrial fibrillation: a prospective observational single centre study. Eur Heart J 2009;30(6):699-709. 
14. Miyazaki S, Kajiyama T, Watanabe T, et al. Impact of electrical connections between ipsilateral pulmonary veins on the second-generation cryoballoon ablation procedure. J Cardiovasc Electrophysiol 2018;30(1):27-31.

15. Okishige K, Aoyagi H, Kawaguchi N, et al. Novel method for earlier detection of phrenic nerve injury during cryoballoon applications for electrical isolation of pulmonary veins in patients with atrial fibrillation. Heart Rhythm 2016;13(9):1810-1816.

16. Perez-Castellano N, Villacastin J, Salinas J, et al. Epicardial connections between the pulmonary veins and left atrium: relevance for atrial fibrillation ablation. J Cardiovasc Electrophysiol 2011;22(2):149-159.

17. Barrio-Lopez MT, Sanchez-Quintana D, Garcia-Martinez J, et al. Epicardial connections involving pulmonary veins: the prevalence, predictors, and implications for ablation outcome. Circ Arrhythm Electrophysiol 2020;13(1):e007544.

18. Yoshida K, Baba M, Shinoda Y, et al. Epicardial connection between the right-sided pulmonary venous carina and the right atrium in patients with atrial fibrillation: a possible mechanism for preclusion of pulmonary vein isolation without carina ablation. Heart Rhythm 2019;16(5):671-678.

19. Tritto M, De Ponti R, Zardini M, Spadacini G, Oliveira M, Salerno-Uriarte JA. Electrical connections between pulmonary veins in humans: evidence after radiofrequency ablation of the venoatrial junction. Circulation 2001;104(7):E30-E31.

20. Takahashi A. Electrical connections between pulmonary veins: implication for ostial ablation of pulmonary veins in patients with paroxysmal atrial fibrillation. Circulation 2002;105(25):2998-3003.

21. Matsuo S, Yamane T, Tokuda M, et al. The dormant epicardial reconnection of pulmonary vein: an unusual cause of recurrent atrial fibrillation after pulmonary vein isolation. Pacing Clin Electrophysiol 2008;31(7):920-924.

22. Mulder MJ, Kemme MJB, Gotte MJW, et al. Differences between gap-related persistent conduction and carina-related persistent conduction during radiofrequency pulmonary vein isolation. J Cardiovasc Electrophysiol 2020;31(7):1616-1627.

23. Ho SY, Cabrera JA, Tran VH, Farre J, Anderson RH, Sanchez-Quintana D. Architecture of the pulmonary veins: relevance to radiofrequency ablation. Heart 2001;86(3):265-270.

24. Cabrera JA, Ho SY, Climent V, Fuertes B, Murillo M, Sanchez-Quintana D. Morphological evidence of muscular connections between contiguous pulmonary venous orifices: relevance of the interpulmonary isthmus for catheter ablation in atrial fibrillation. Heart Rhythm 2009;6(8):1192-1198.

\section{FIGURES}

FIGURE 1 Patient distribution flowchart. The nadir balloon temperature and balloon occlusion status were analyzed and compared among the three groups. CB, cryoballoon; PV, pulmonary vein

\section{Hosted file}

image1.emf available at https://authorea.com/users/349556/articles/474545-predictors-of-asuccessful-crosstalk-ablation-technique-during-second-generation-cryoballoon-ablationin-patients-with-atrial-fibrillation

FIGURE 2 Comparison of factors during ablation among the three groups. A, The average NT was significantly lower in the CST success group than in the CST failure group. B, The percentage of complete occlusion of the superior PV with CB was significantly higher in the CST success group than in the other groups*. 95\% confidence intervals are shown in (A), whereas $98.3 \%$ confidence intervals are shown in (B). *: $\mathrm{p}<0.0167$ was considered significant, with adjustment by Bonferroni correction. CB, cryoballoon; CST, crosstalk; NT, nadir balloon temperature; PV, pulmonary vein 
(B)

\section{Hosted file}

image2.emf available at https://authorea.com/users/349556/articles/474545-predictors-of-asuccessful-crosstalk-ablation-technique-during-second-generation-cryoballoon-ablationin-patients-with-atrial-fibrillation

\section{Hosted file}

image3.emf available at https://authorea.com/users/349556/articles/474545-predictors-of-asuccessful-crosstalk-ablation-technique-during-second-generation-cryoballoon-ablationin-patients-with-atrial-fibrillation

FIGURE 3 Distribution of the NT and the occlusion status of the superior PV among patients in the CST ablation success group and the CST ablation failure group. The NT in the CST ablation success group was mainly observed in the temperature range of [?]-46degC when complete occlusion was achieved. In contrast, the NT in the CST ablation failure group was widely scattered throughout all temperatures, except in temperatures in which the CST ablation success group was mainly observed. CST, crosstalk; NT, nadir balloon temperature; PV, pulmonary vein

FIGURE 4 Successful CST ablation case. A, LSPV isolation was not achieved by CB ablation with an NT of $52 \mathrm{deg}$ C, complete LSPV occlusion, and freezing duration of 240 seconds. B, Successful CST ablation during CB ablation of the LIPV was observed. Both LSPV and LIPV were isolated simultaneously and LIPV ectopic beats conducted to the LSPV without conducting to the LA. AP, anterior posterior; CB, cryoballoon; CS, coronary sinus; CST, crosstalk; LA, left atrium; LAO, left anterior oblique; LSPV, left superior pulmonary vein; LIPV, left inferior pulmonary vein; NT, nadir balloon temperature

\section{(A)}

\section{Hosted file}

image5.emf available at https://authorea.com/users/349556/articles/474545-predictors-of-asuccessful-crosstalk-ablation-technique-during-second-generation-cryoballoon-ablationin-patients-with-atrial-fibrillation

FIGURE 5 The speculated mechanisms of CST phenomenon. There are two possible mechanisms for the CST phenomenon. CB, cryoballoon; CST, crosstalk; PV, pulmonary vein

\section{Hosted file}

image7.emf available at https://authorea.com/users/349556/articles/474545-predictors-of-asuccessful-crosstalk-ablation-technique-during-second-generation-cryoballoon-ablationin-patients-with-atrial-fibrillation

\section{TABLES}

TABLE 1 Baseline clinical characteristics

\section{Hosted file}

image8.emf available at https://authorea.com/users/349556/articles/474545-predictors-of-asuccessful-crosstalk-ablation-technique-during-second-generation-cryoballoon-ablationin-patients-with-atrial-fibrillation

Abbreviations: AF, atrial fibrillation; LA, left atrium; LV, left ventricle 
TABLE 2 Characteristics of PVs in the CST success group

\title{
Hosted file
}

\begin{abstract}
image9.emf available at https://authorea.com/users/349556/articles/474545-predictors-of-asuccessful-crosstalk-ablation-technique-during-second-generation-cryoballoon-ablationin-patients-with-atrial-fibrillation
\end{abstract}

Abbreviations: CB, cryoballoon; LS, left superior; NA, not available; NR, not recorded during cryoballoon application; NT, nadir balloon temperature; PV, pulmonary vein; PVI, pulmonary vein isolation; RS, right superior 